\title{
Belphégor
}

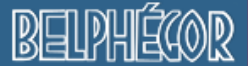

Littérature populaire et culture médiatique

13-1 | 2015

Distinctions That Matter/Fictions Économiques

\section{Corto Maltese tra fumetto e letteratura disegnata}

\section{Giovanni Remonato}

\section{(2) OpenEdition}

\section{Journals}

\section{Edizione digitale}

URL: https://journals.openedition.org/belphegor/620

DOI: $10.4000 /$ belphegor.620

ISSN: 1499-7185

Editore

LPCM

\section{Notizia bibliografica digitale}

Giovanni Remonato, «Corto Maltese tra fumetto e letteratura disegnata », Belphégor [En ligne], 13-1 | 2015, mis en ligne le 02 juin 2015, consulté le 21 septembre 2021. URL : http://

journals.openedition.org/belphegor/620; DOI : https://doi.org/10.4000/belphegor.620

Questo documento è stato generato automaticamente il 21 septembre 2021.

\section{(c) (i) (9)}

Belphégor est mis à disposition selon les termes de la Licence Creative Commons Attribution - Pas d'Utilisation Commerciale - Pas de Modification 4.0 International. 


\title{
Corto Maltese tra fumetto e letteratura disegnata
}

\author{
Giovanni Remonato
}

\section{L'avventura editoriale}

1 Corto Maltese nacque dalla matita di Hugo Pratt nel luglio del 1967, quando nella rivista Sgt. Kirk venne pubblicata la prima puntata di Una ballata del mare salato. Questo fumetto è un lungo racconto corale auto-conclusivo e nei piani del suo autore doveva restare un episodio a sé stante. Hugo Pratt non poteva ancora sapere che quel marinaio, nato sull'isola di Malta, sarebbe stato il suo personaggio di maggior successo, che l'avrebbe accompagnato per tutta la vita. Nello stesso anno Hugo Pratt incontrò a Parigi Georges Rieu, editore di Pif Gadget, che gli propose di realizzare dei fumetti per la sua rivista che avessero come protagonista proprio quel marinaio di Una ballata del mare salato. Nell'Aprile del 1970 Corto Maltese riapparve in un nuovo episodio: Il segreto di Tristan Bantam. Tra il 1970 e il 1973 uscirono per Pif Gadget ventuno fumetti, tutti con al centro la figura di Corto Maltese.

2 Il successo delle storie di Corto Maltese dalla Francia si propagò in Italia e poi in tutta Europa. In Italia i fumetti di Corto Maltese pubblicati in Francia da Pif furono tradotti e riproposti dalle riviste più prestigiose. Dapprima nel Corriere dei Piccoli, mentre, a partire dal 1972, le avventure di Corto Maltese si poterono leggere su Linus. Nel 1974 Linus iniziò la pubblicazione di Corte sconta detta arcana, che segnò il ritorno a una narrazione di ampio respiro, più complessa e articolata. Infine, nel 1980, vennero pubblicate le prime ventidue tavole di La casa dorata di Samarcanda. Nel 1977 uscì Favola di Venezia (o Sirat al Bunduqiyyah) a colori (colori di Mariolina Pasqualini) per il settimanale L'Europeo. Le rimanenti avventure di Corto Maltese, a cominciare dalla già citata La casa dorata di Samarcanda, si trasferirono in seguito sull'omonima rivista, che venne fondata nel 1983. Unica eccezione fu La Giovinezza, che venne pubblicata in bianco e nero a partire dal 1981 nel quotidiano francese Le Matin de Paris. Corto Maltese non si limitò però al solo fumetto. Nel 1995 Hugo Pratt pubblicò per Einaudi il romanzo Corto Maltese. Una ballata del mare 
salato, novelization dell'omonimo fumetto. Con lo stesso procedimento nel 1996 venne pubblicato postumo, a cura di Marco Steiner, Corto Maltese. Corte Sconta detta arcana, sempre per i tipi di Einaudi. ${ }^{1}$

3 Con la scomparsa di Hugo Pratt, avvenuta nel 1995, si interruppero anche le avventure del marinaio di sua creazione. Il successo dei suoi fumetti non è però diminuito, come dimostrano le numerose ristampe, le trasposizioni per il cinema e la televisione, le mostre in diversi musei e le più disparate creazioni che si sono inspirate alla figura di Corto Maltese.

\section{Le riviste}

Hugo Pratt fu notoriamente sempre molto diffidente nei riguardi degli editori. Tuttavia, grazie al successo di Corto Maltese, divenne molto famoso, forse uno degli autori di fumetto più celebri dell'epoca. I suoi fumetti venivano contesi dalle varie riviste e case editrici. Negli anni in cui Corto Maltese si affermava come uno dei personaggi più amati, il fumetto andava incontro a profondi cambiamenti, anche dal punto di vista editoriale. Hugo Pratt seppe sfruttare al meglio questi cambiamenti, riuscendo a trovare nuove e sempre più prestigiose soluzioni per pubblicare i suoi fumetti.

Dopo le primissime esperienze editoriali a Venezia nel dopoguerra, Hugo Pratt ebbe una proposta di lavoro in Argentina dove, per un lungo periodo, poté fare esperienza con alcuni tra i più grandi disegnatori e sceneggiatori di quei tempi come Héctor Germán Oesterheld. ${ }^{2}$ Tornato in Italia Hugo Pratt lavorò al Corriere dei Piccoli. Questa celebre rivista nacque nel 1908 come supplemento al Corriere della Sera, sotto la direzione di Silvio Spaventa Filippi: 'Il gioco consiste nel mettere in mano al bambino un 'vero' giornale - e la testata richiama quella normalmente acquistata e letta dal papà - ma è anche un giornalino pensato e realizzato solo per loro". ${ }^{3}$ Il Corriere dei Piccoli ebbe il grande merito di lanciare il fumetto in Italia anche se, soprattutto nei primi anni, veniva trattato con una certa cautela, in quanto in certi ambienti veniva ritenuto diseducativo. Ecco quindi che le vignette venivano adattate e modificate secondo i gusti e le esigenze pedagogiche del giornale eliminando, ad esempio, le nuvolette e spostando il testo in didascalia: "Nasce quindi la prima testata a fumetti italiana e, insieme, il primo fumetto di autore italiano, con una sola pecca: i 'fumetti', cioè le nuvolette con le parole dei dialoghi, non ci sono (sostituite da didascalie in rima)." I testi dei balloon venivano adattati in didascalia in forma di strofe di ottonari in rima baciata non per scelte grafiche, ma perché dovevano educare i ragazzi alla metrica della poesia e alla lingua della prosa e, in nessun caso, imitare il linguaggio parlato.

6 Nel primo numero (figura 1) l'ultima striscia si concludeva con il messaggio: "Chi vuol fare l'altrui danno/ha le beffe ed ha il malanno". Il carattere educativo della testata era ribadito dal fatto che ai fumetti si alternavano alcuni articoli divulgativi, brevi testi di prosa o di teatro, poesie e giochi pensati apposta per i ragazzi. Il primi numeri, ad uscita settimanale, avevano un prezzo di dieci centesimi di Lire, un formato giornale $(22,5 \times 30 \mathrm{~cm})$ con sovraccoperta (tranne il primo numero), venti pagine (i primi due numeri, poi sedici) a quattro colori e in bianco e nero. Hugo Pratt realizzò per il popolare giornale per ragazzi alcune illustrazioni e trasposizioni a fumetti di romanzi di avventura come L'isola del tesoro e Il ragazzo rapito di Robert Louis Stevenson (testi di Mino Milani) o di classici come L'Odissea (sceneggiatura di Franco Basaglia). Sulle pagine del Corriere dei 
Piccoli venne stampato il primo vero fumetto scritto e disegnato completamente da Hugo Pratt: Anna nella giungla, che era già stato pubblicato in Argentina.

Figura 1

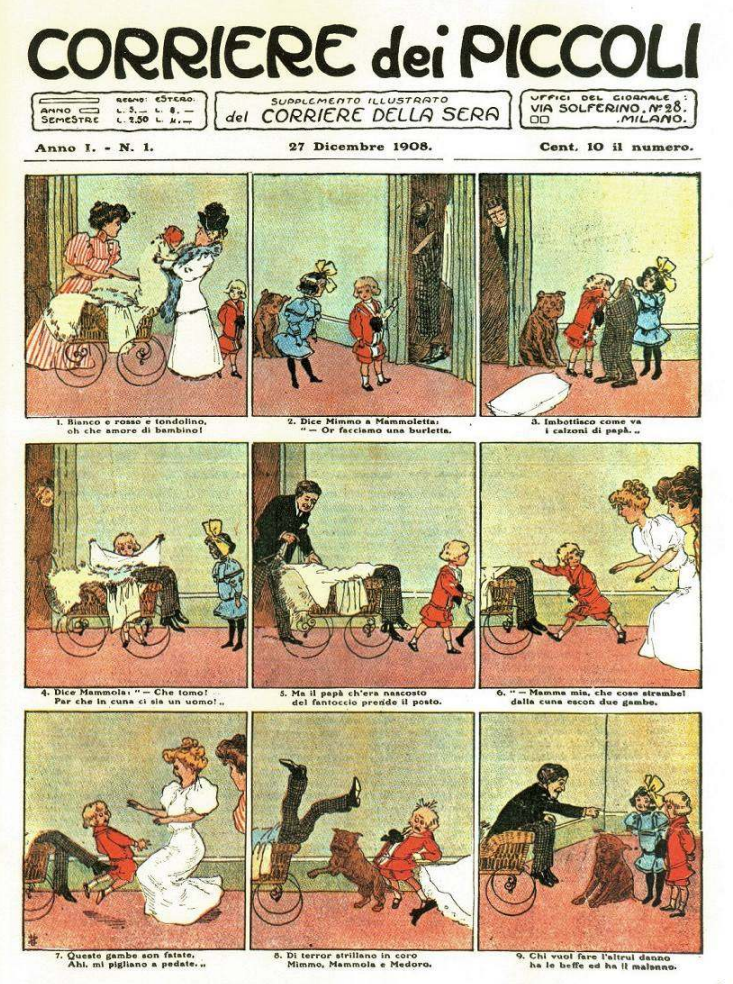

|l Corriere dei Piccoli, n.1

In cerca di sempre maggiore libertà per le sue storie, Hugo Pratt lasciò temporaneamente il Corriere dei Piccoli e accettò la proposta dell'editore genovese Florenzo Ivaldi di collaborare alla realizzazione di una nuova rivista di fumetti che si chiamerà Sgt. Kirk, Mensile dei Comics (figura 2). Il nome deriva dal protagonista di un fumetto di Pratt realiazzato in Argentina (testi di Oesterheld). Ivaldi volle creare un contenitore per fumetti che rimettesse al centro l'avventura, dopo che riviste come Linus avevano puntato sulla satira e sul fumetto engagé. La grafica della copertina era curata dallo stesso Pratt, che disegnò personalmente sia il logo, sia alcune immagini di sfondo (tutte a colori). I fascicoli di Sgt. Kirk erano molto eleganti e venivano stampati su carta di grande qualità. Eleganza e qualità avevano però una controindicazione: il prezzo di copertina relativamente alto, 500 Lire, a fronte delle 300 di Linus. Il costo della rivista fu forse una delle cause della prematura chiusura di Sgt. Kirk. L'esperienza di Hugo Pratt a Sgt. Kirk fu di centrale importanza, in quanto rappresentò il primo contatto dell'autore con una rivista specializzata in fumetti. Questo tipo di riviste rappresentarono un'evoluzione rispetto alle pubblicazioni per ragazzi tipo il Corriere dei Piccoli sia per la forma editoriale e per la qualità dei materiali usati, sia per il ruolo centrale che svolgeva il fumetto al loro interno. Oltretutto Hugo Pratt godette a Sgt. Kirk di una grande libertà espressiva, cosa piuttosto rara per un autore che all'epoca era pressoché sconosciuto, almeno in Italia. Grazie al generoso sostegno di Florenzo Ivaldi, Hugo Pratt poté concepire e realizzare il suo primo grande successo, Una ballata del mare salato, un fumetto che, sotto tutti i punti di vista, rappresentò una grande novità per l'epoca. 


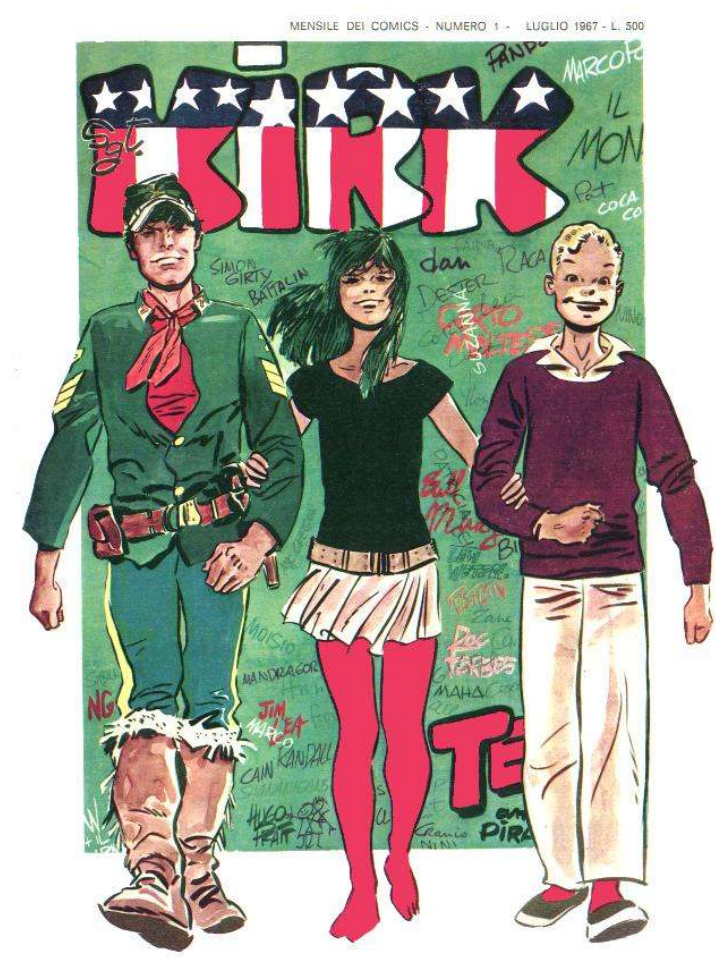

Sgt Kirk, n.1 francese Pif, che in quegli anni aveva un notevole successo di vendite. Raggiunto il successo in Francia, Hugo Pratt si accordò con il Corriere dei Piccoli per ripubblicare Una ballata del mare salato nel famoso giornale per ragazzi, facendo così conoscere il suo primo lavoro al grande pubblico e sancendo definitivamente il successo di Corto Maltese anche in Italia.

Il passaggio successivo avvenne nel 1972, quando Corto Maltese si trasferì su Linus, la rivista italiana di fumetti di maggior prestigio (figura 3). Molti la definiscono addirittura la prima rivista di fumetti del mondo. ${ }^{5}$ La rivista nacque nel 1965 sotto la direzione di Giovanni Gandini come mensile per i tipi di Milano Libri. Linus ebbe un'importanza straordinaria, non solo per aver fatto scoprire al pubblico italiano i migliori fumetti di tutto il mondo e per aver lanciato molti nuovi autori; uno dei meriti principali della rivista fu quello di aver sdoganato il fumetto, svecchiandone l'immagine che lo voleva retaggio esclusivo di un pubblico giovane o letterariamente non maturo. Il fumetto era, per i redattori di Linus, una cosa seria da trattare come un vero prodotto di cultura. Ecco cosa si leggeva in una sorta di editoriale programmatico nel primo numero:

Questa rivista è dedicata per intero ai fumetti. Fumetti s'intende di buona qualità, ma senza pregiudizi intellettualistici. Accanto alle storie e ai personaggi più moderni e significativi come i 'Peanuts' (studiati ormai come un autentico prodotto di cultura), la rivista intende presentare fumetti di avventura, classici per l'infanzia, inediti di giovani autori. L'unico criterio di scelta di questa 'letteratura grafica' è quello del valore delle singole opere, del divertimento che ne può trarre il lettore, oggi; non quello di un interesse puramente documentario o archeologico. I classici della storia del fumetto che pubblicheremo saranno solo quelli veramente originali 
e ancora validi oggi, verificati a una lettura il più possibile disinteressata, scevra di mitologie. Cercheremo poi di presentare al pubblico italiano quei fumetti che ancora non conosce, di rivelargli tempestivamente le nuove scoperte di tutto il mondo, di tenerlo informato su quanto avviene e si dice in questo campo. ${ }^{6}$

Figura 3

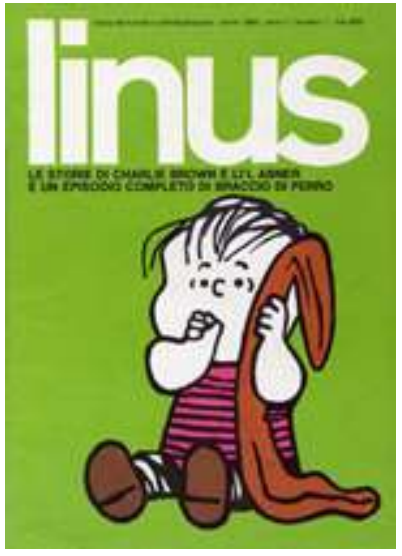

Linus, n.1

Interessante è questo precoce uso della definizione letteratura grafica, che anticipa di molti anni la famosa dicitura di Will Eisner "graphic novel". Linus cercava insomma di emancipare il fumetto accostandolo al molto più accreditato mondo della letteratura. $\mathrm{A}$ sostenere questa operazione intervennero delle personalità che il quel campo, quello della letteratura, erano tra le massime autorità dell'epoca: Umberto Eco, Elio Vittorini, Oreste Del Buono e Vittorio Spinazzola. Nel primo numero si poteva leggere un'intervista sui Peanuts di Umberto Eco a Oreste Del Buono e a Elio Vittorini, in cui quest'ultimo, tra le altre cose, affermava: "Bisognerebbe prima stabilire a che tipo di letteratura appartiene Schulz, ma comunque, senza andare nel difficile, io lo avvicinerei a Salinger, però con un interesse molto più ampio e secondo me molto più profondo".

11 Linus abbandonò il formato "giornale" del Corriere dei Piccoli e, congruamente con la linea editoriale, optò per un formato "rivista" $(20.5 \times 27 \mathrm{~cm})$ con sessantaquattro pagine in bianco e nero. Il formato editoriale, così come la cura della grafica e dei materiali di stampa furono fattori decisivi per il successo di Linus. La copertina, curata da Salvatore Gregoretti, ha fatto scuola nel mondo delle riviste per la sua nitidezza, con il titolo generalmente bianco e uno sfondo monocromatico su cui si staglia una figura unica, di solito un personaggio di Schulz.

La pubblicazione dei fumetti di Corto Maltese su Linus continuò fino al 1983 quando il gruppo editoriale Rizzoli decise di sfruttare la grandissima notorietà del marinaio di Hugo Pratt usando il suo nome come titolo per una nuova rivista di fumetti. Nacque così Corto Maltese (figura 4), la rivista. Corto Maltese metteva in risalto, ovviamente, ai fumetti di Hugo Pratt, ma si potevano leggere anche altri bravissimi autori come Andrea Pazienza, Milo Manara, Moebius, Frank Miller e Alan Moore. La rivista vantava una veste grafica molto elegante $(22 \times 29 \mathrm{~cm})$ a cura di Salvatore Gregorietti, lo stesso che aveva ideato la grafica di Linus. La copertina era formata dal titolo e dai principali contenuti in testa, mentre una figura occupava tutto lo spazio restante su sfondo monocromatico. La rivista era composta da centoquattordici pagine spillate, a colori e con una spessa carta patinata. 


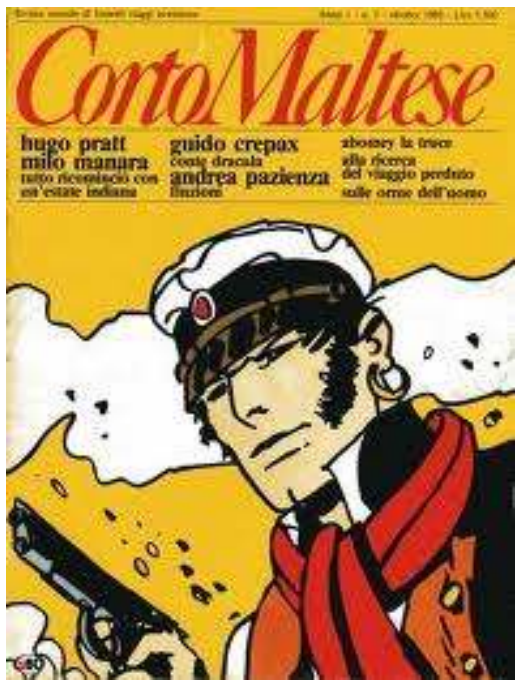

Corto Maltese, n. 1

\section{Dalle riviste al libro}

13 Le pubblicazioni della rivista Corto Maltese continuarono fino al 1993, nonostante le continue perdite: “per la rivista non c'è mai stata una chiusura in attivo (a parte quella dei primi tre numeri)". ${ }^{8}$ Con l'ultimo numero di Corto Maltese si chiuse forse un'epoca: quella delle grandi riviste del fumetto che, per alcuni anni, diedero grande risalto a questo mezzo espressivo. Con la crisi del modello editoriale delle riviste contenitrici specializzate, il fumetto dovette trovare nuove strade per essere pubblicato e letto. Una delle ultime tendenze è quella di orientarsi sempre più al modello della letteratura, in particolare al romanzo. Questo nuovo fumetto abbandona la serialità in favore di una concezione unitaria del racconto, che viene programmato fin da subito per la pubblicazione in volume. Il fumetto nelle librerie passa così dallo scaffale delle letture per l'infanzia a quello della letteratura di varia e in questo passaggio viene ribattezzato graphic novel, Il termine è notoriamente stato introdotto da Will Eisner nel 1978 quando, proprio per sottolineare la perdita del carattere comico che contraddistingueva i suoi fumetti, volle apporre questa etichetta al suo A Contract with God in sostituzione della classica dicitura comics. ${ }^{9}$

In questo processo evolutivo che ha investito il mondo del fumetto, Hugo Pratt è stato un precursore. Non solo egli seppe inserire i suoi fumetti in riviste sempre meno infantili e culturalmente più intraprendenti. Fu anche uno dei primi autori di fumetto che vide le sue opere pubblicate in volume, un formato che mima espressamente la forma editoriale con cui si pubblicano romanzi, poesie, ecc.; in poche parole la letteratura vera e propria. Nel 1972, contemporaneamente all'uscita su Linus, la casa editrice Mondadori pubblicò nella collana L'Intrepida una versione in volume unico cartonato di Una ballata del mare salato, in bianco e nero in formato verticale $(19.5 \times 26,5 \mathrm{~cm})$. Curiosamente in questa versione il titolo venne cambiato in: La ballata del mare salato (figura 5). Molto curata è l'edizione del 1976 dello stesso fumetto di una piccola casa editrice, Albatros, che pubblicò un volume di lusso cartonato con sovraccoperta trasparente $(25 \times 35 \mathrm{~cm})$. Questa fu anche la prima edizione a colori di Una ballata del mare salato (colori di Mariolina Pasqualini) e 
venne arricchita da una serie di quindici acquerelli di Pratt intitolati: Corto Maltese raccontato da Corto Maltese.

Figura 5

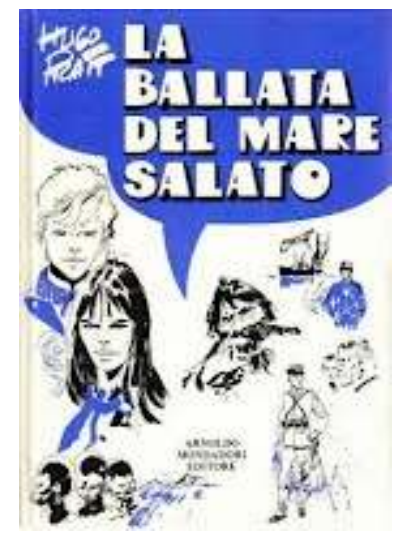

La ballata del mare salato

La prima vera edizione in volume dei fumetti di Corto Maltese è però francese: nel 1971 la casa editrice Publicness pubblicò una raccolta in due volumi cartonati con formato orizzontale all'italiana, $(33 \times 24 \mathrm{~cm})$ e in bianco e nero di alcuni episodi usciti precedentemente sulla rivista Pif. Il primo volume, edito per celebrare l'assegnazione al maestro veneziano del prestigioso premio Phenix per il miglior fumetto d'avventura, raccoglie i primi sei episodi e ha una sovraccoperta a sfondo marrone, che nasconde una coperta a sfondo bianco. Di questo volume esiste anche un'edizione limitata (un tirage de tête di duecentocinquanta esemplari) con coperta telata. Stando alle indicazioni di una casa d'asta torinese, il valore di questa edizione di lusso si aggira oggi tra i duemilaottocento e i seimila euro..$^{10} \mathrm{Il}$ secondo volume, del 1974, raccoglie altri cinque episodi (colorati da Anne Froigner). Questo volume venne pubblicato nello stesso anno anche in Italia da Milano Libri, con una tiratura limitata a cinquemila esemplari. La versione italiana comprende anche la serie di acquerelli realizzati da Pratt Un Oroscopo per Corto Maltese. Anche la prima edizione integrale del ciclo di Corto Maltese fu stampata in Francia. La pubblicazione iniziò nel 1975 sotto la supervisione dello stesso Pratt per i tipi di Casterman.

Le ristampe dei fumetti di Pratt in formato volume sono numerose. Recentemente i fumetti del padre di Corto Maltese hanno superato perfino i confini delle librerie, arrivando a essere distribuiti con i quotidiani in edicola. Le testate italiane sono solite proporre allegati di vario genere (film, libri, gadget vari) per incentivare la vendita dei giornali. Nel 2003 il gruppo editoriale L'Espresso, in collaborazione con Panini Comics, distribuì, in allegato ai giornali del gruppo, una nuova serie: I Classici del fumetto di Repubblica. La serie si proponeva di raccogliere in trenta volumi tascabili (brossurati con alette) il meglio del fumetto internazionale. Il primo volume, distribuito gratuitamente, fu dedicato proprio a Hugo Pratt e raccoglieva Una ballata del mare salato in bianco e nero e Favola di Venezia con i colori di Patrizia Zanotti.

L'operazione più pregevole è quella del 2006, quando lo stesso gruppo editoriale distribuì tutta la serie di Corto Maltese. Questa edizione ripropone l'opera di Pratt in dieci volumi cartonati di grandi dimensioni $(21 \times 29 \mathrm{~cm})$ con carta plastificata di grande qualità. Un'opera insomma di pregevole fattura dal punto di vista dei materiali e con un prezzo di 
vendita competitivo (nove euro e novanta, costo del giornale incluso). Il pregio di questi volumi non sta soltanto nella grande qualità dei materiali, ma anche nell'ampio apparato critico che lo accompagna. Ogni volume contiene infatti una lunga introduzione in cui si susseguono approfondimenti sul personaggio o sulla saga di Corto Maltese (a cura di Oscar Cosulich, Luca Boschi e Sergio Rossi). Viene sempre fornito un quadro storico di riferimento e una carta geografica indica i luoghi delle avventure. Vi sono poi interventi di personalità varie del mondo della cultura invitate a esprimersi su di un particolare aspetto dei fumetti di Pratt. Si possono leggere articoli di critici letterari (Umberto Eco), registi (Gabriele Salvatores, Pascal Morelli, Enzo D’Alò), attori (Valerio Mastandrea) cantautori (Gino Paoli), autori di fumetto e illustratori (Milo Manara, Silvia Ziche, Massimo Bucchi), ecc. L'iniziativa del gruppo L'Espresso si può quindi leggere come un tentativo di ribadire ancora una volta il valore canonico dell'opera di Pratt. In effetti nessun altro autore di fumetto aveva ricevuto così grande attenzione da parte dei quotidiani italiani, che solitamente riservano questo tipo di iniziative ai grandi classici della letteratura. Da allora le pubblicazioni dell'opera di Pratt in allegato ai quotidiani sono continuate. Da segnalare l'edizione di Una ballata del mare salato sul sito del Corriere della Sera. ${ }^{11}$ La versione online è accessibile gratuitamente e serviva a lanciare una nuova edizione dei fumetti di Pratt in versione ebook. ${ }^{12}$

\section{Letterarietà di Corto Maltese}

18 I fumetti di Hugo Pratt mossero i primi passi negli anni Sessanta, anni di grandi novità nel mondo del fumetto, non solo dal punto di vista editoriale. In Italia, come negli Stati Uniti e in Francia, gli autori di fumetto esplorarono nuovi generi, inventarono nuovi stili. Nacquero così fumetti, che ruppero gli schemi del classico fumetto d'avventura o comico, affrontando temi sempre più complessi come la violenza, il crimine, ma anche il sesso, la droga, la critica sociale e la contestazione politica. Anche i personaggi cominciarono a differenziarsi, incorporando valori non sempre positivi o, a volte, del tutto negativi: "sono i personaggi cosiddetti 'neri' (Diabolik, Kriminal, Satanik) ad attirare l'interesse del pubblico adulto: il successo incontrato dalle avventure degli eroi neri rese evidente il fatto che il fumetto aveva un mercato potenziale assai ampio, che toccava, oltre ai ragazzini, anche i giovani e gli adulti". ${ }^{13}$

\section{Un uomo del destino}

Quando nel 1967 Hugo Pratt accettò di collaborare con l'editore Ivaldi ad una nuova rivista per i suoi fumetti, lo fece per poter creare le sue storie con maggior libertà. I lettori che nel 1967 affrontarono la nuova produzione del fumettista veneziano su Sgt. Kirk, si trovarono di fronte ad un fumetto del tutto nuovo, lontano dai generi comico o avventuroso che dominavano il mercato del fumetto: "per gli adolescenti abituati agli strampalati, divertentissimi fumetti umoristici di Cocco Bill e Zorry Kid, a quelli di Lucky Luke oppure alle realistiche avventure di Dan Cooper e Michel Vaillant, era una sorpresa piena di novità". ${ }^{14}$ Vestito sempre elegantemente, con una punta di finta trascuratezza, capelli ricci e arruffati nascosti sotto un cappello da marinaio, "sguardo tagliente come una lama d'acciaio", ${ }^{15}$ viso segnato dal sole e dalle molte avventure vissute, orecchino a ricordarci il suo legame con il mare, il personaggio di Corto Maltese non evocava nessuno 
dei tanti eroi protagonisti dei fumetti degli anni sessanta, che per la maggior parte si ispiravano ai modelli americani:

Corto non è integerrimo come Dick Tracy, mascherato come Phantom o Diabolik; non è ariano puro come Flash Gordon, provinciale come Li'l Abner, muscoloso e manesco come Dick Fulmine. È privo di poteri o superpoteri, e non solo di quelli tipici dell'alieno o del fenomeno della natura, ma persino di quelli più terreni, ipnotici, che spesso tolgono d'impaccio il mago Mandrake. Non ha amici o 'spalle' su cui contare, né gorilla come Lothar, né buffi fornitori di battute, tanto meno ragazzini apprendisti di bottega. ${ }^{16}$

Hugo Pratt cercò insomma di creare un personaggio di impronta mediterranea, "per fare qualcosa di diverso dagli eroi di avventura anglosassoni". ${ }^{17}$ La madre è una gitana di Gibilterra, amante del rabbino di Cordova e di Malta, il padre è un marinaio della Cornovaglia. Corto Maltese è quindi abituato a confrontarsi con tradizioni e culture diverse e si trova a suo agio in ogni paese in cui si viene a trovare: "L'aplomb del Maltese è il risultato della sua cultura, del suo girovagare per il mondo, dell'elaborazione della conoscenza". ${ }^{18}$ Questa mescolanza di origini ricorda un celebre personaggio della letteratura che porta anche un nome simile: il Kurtz di Heart of Darkness a cui Conrad assegnò un antenato in pressoché ogni stato europeo..$^{19}$ Hugo Pratt propose invece, in numerose interviste, un'etimologia diversa per il nome del suo personaggio: "in argot spagnolo, 'corto' vuol dire 'svelto di mano', 'ladro' - ma anche 'ladro di sentimenti' - e 'Maltese' lo situa bene in un panorama mediterraneo; all'epoca si parlava molto dell'isola di Malta, che aveva ottenuto l'indipendenza solo da pochi anni". ${ }^{20}$

Che Corto sia un eroe per-i-fatti-suoi, appare chiaro fin dal suo primo ingresso in scena, che non è certo dei più gloriosi. In Una ballata del mare salato lo troviamo infatti legato ad una croce su una zattera alla deriva. Veste solo i pantaloni e ha la barba lunga, come si vede nella figura 6. Oltretutto non gode nemmeno del privilegio di aprire il racconto, visto che le prime vignette sono dedicate all'oceano, che reclama per sé il ruolo di vero protagonista della vicenda. Anche da questi particolari, dal suo esordio da semplice comprimario, Corto Maltese si rivela come personaggio che fatica ad accettare il ruolo di eroe protagonista. Corto non è nemmeno un eroe senza macchia e senza paura. In Una ballata del mare salato appare fragile tanto che, nel corso della vicenda, rischierà di morire più volte. In effetti sembra che egli prenda parte a queste avventure suo malgrado. Preferirebbe starsene per i fatti suoi, magari a leggere un libro. Riesce a vincere il distacco quasi apatico che lo contraddistingue solo con una certa riluttanza. In Nonni e fiabe risponde a un indigeno che gli chiede il nome in questo modo: "Corto ... Corto Maltese ... Ma visto che gli indios hanno nomi segreti ... Puoi chiamarmi Uno-che-nonriesce-mai-a-starsene-per-i-fatti- suoi". Anche Cranio in Una ballata del mare salato ne parla in modo simile: "Non ha patria ed è un uomo libero che sa molte cose ... Ma non vuole prendersi responsabilità". 


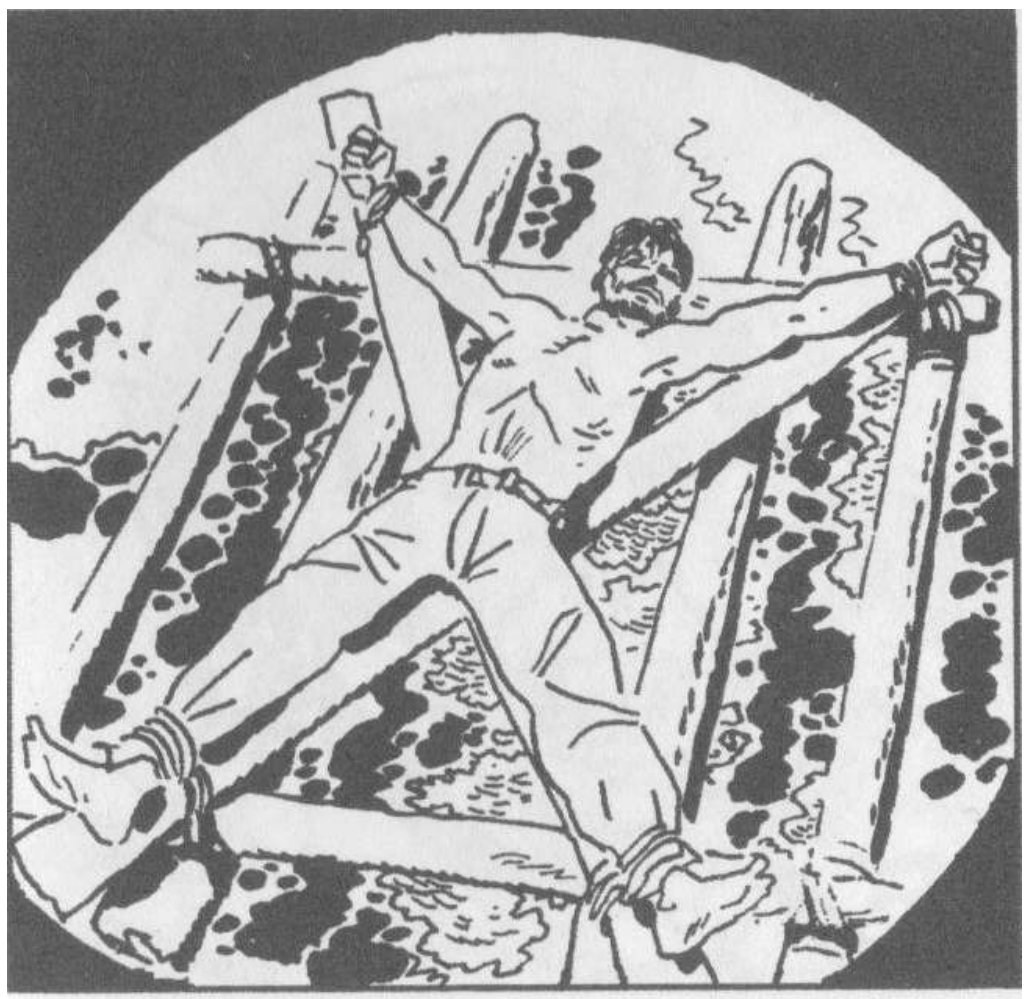

"Non c'è dubbio, è Corto Maltese"

Non è nemmeno per un senso di giustizia o per un innato altruismo che Corto Maltese sceglie di impegnarsi in una nuova impresa. Quando Tristan Bantam in Un'aquila nella giungla esclama sicuro: "Se sono tedeschi sono nemici !", Corto Maltese lo frena dicendo: "Non ho nemici io! Mi faccio gli affari miei e basta". In Nonni e fiabe dopo una violenta colluttazione con un certo Mendoza, ci tiene a precisare che lo ha fatto non perché questo Mendoza è uno schiavista ma perché ha tentato di imbrogliarlo ai dadi: "Non mi importa cosa faccia questo Mendoza. Non sono affari miei". Hugo Pratt ci teneva a sottolineare questa dubbia moralità di Corto Maltese e propose una lettura inquietante della già citata entrata in scena con il marinaio legato ad una croce in Una ballata del mare salato: "la situazione di Corto Maltese disteso su quella zattera non è inverosimile: quel tipo di crocifissione veniva praticata come punizione presso alcuni popoli del Pacifico, per esempio in caso di stupro. Con questo non voglio dire che Corto Maltese abbia veramente violentato un'indigena, anche lui ha i suoi segreti e lei sa a che punto io rispetti la sua vita privata". ${ }^{21}$

Corto Maltese è dunque un personaggio sfuggente, ambiguo fino alla contraddizione, complesso e difficilmente inquadrabile, insomma un vero personaggio a tutto tondo. In questo Hugo Pratt dimostra di aver saputo creare una nuova sintesi tra i protagonisti del fumetto d'avventura alla Tarzan o alla Prince Valiant e gli eroi negativi dei neri italiani come Diabolik creando un personaggio che, secondo Herbert Heinzelmann, si avvicina alla figura dell' eroe post-moderno della letteratura del novecento:

Der Held ist nicht mehr das, was ihn stets als solchen kennzeichnete : ein Täter, ein (moralisch) Agierender, die handelnde (oder erleidende) Kraft. Oft ist er zur Tat nicht mehr fähig, ist eigenschaftslos (bei Musil), eine Maske für den Mythos (bei Joyce) oder ein Zahnrad in einem größeren Mechanismus wie der Stadt (bei Döblin). 
Die Autoren verlieren den göttlichen Einblick in seine Gedanken und Motive. Sie müssen nach ihm recherchieren, er bleibt ihnen fremd, ein Anonymus, den fast zufällig das Interesse streift. Genau so ein Held ist Corto Maltese in Hugo Pratts Südseeballade. Und genau so einen Held gab es bis dahin in den Comics nicht. ${ }^{22}$ di Hugo Pratt non devono però trarre in inganno. Corto Maltese non è un personaggio di un romanzo che si trova in un fumetto per sbaglio. Il marinaio conserva gelosamente dei tratti meno nobili: Corto è rude, impulsivo, di poche parole, a volte persino macho. Per certi aspetti il Maltese ricorda molto personaggi dei romanzi e film d'avventura. Stando alle dichiarazioni dello stesso Pratt, la vera fonte di ispirazione per il suo personaggio non furono i personaggi di Conrad, né il capitano Nemo, né tantomeno Ahab. Per la silhouette di Corto Maltese sembra che il disegnatore veneziano si sia rifatto all'attore statunitense Burt Lancaster, protagonista di molti film d'avventura. In particolare Pratt prese spunto dal film His Majesty O' Keefe di Byron Haskin in cui un esorbitante Burt Lancaster interpreta un capitano che viene abbandonato dal suo equipaggio e miracolosamente salvato su un'isola dispersa della Micronesia, dove insegnerà agli indigeni a sfruttare le risorse naturali e li difenderà da pirati e trafficanti di schiavi. Infine verrà proclamato re $\mathrm{e}$ rinuncerà a tornare al cosiddetto mondo civile. La somiglianza con la storia e le fattezze di Corto Maltese è tale, che Pratt si rifiutò sempre di trasporre le avventure di Corto Maltese in un film: "E infatti a chi mi chiede un film su Corto Maltese, rispondo che c'è già, basta rovistare fra i fondi di magazzino di qualche cineteca". ${ }^{23}$

\section{Intertestualità prattiane}

Tutta l'opera di Hugo Pratt è disseminata di citazioni e allusioni che rimandano, in modo più o meno diretto, a un vasto repertorio di opere letterarie, miti, film, avvenimenti e personaggi storici ecc. Il continuo richiamo citazionistico rispecchia il gusto e la personalità dell'autore, i cui interessi culturali "spaziavano dal fumetto d'avventura americano - la strip degli anni Trenta e Quaranta - al cinema ; dalla storia alla geografia ; dall'esoterismo alla mitologia ; dai viaggi al vino e alle donne". ${ }^{24}$

Le opere letterarie a cui si fa riferimento nei fumetti della saga di Corto Maltese sono moltissime: si va dai romanzi di avventura di Rudyard Kipling, alle opere dei grandi narratori del mare come Joseph Conrad o Herman Melville; dalle poesie di Arthur Rimbaud a quelle di S.T. Coleridge; dai drammi di William Shakespeare, ai cicli cavallereschi medievali. $\mathrm{A}$ un livello più profondo troviamo allusioni ad opere che hanno fatto da modello, ispirando trame, ambientazioni oppure certi personaggi, fungendo, per dirla con Gérard Genette, da ipotesto. ${ }^{25}$ In Una ballata del mare salato, ad esempio, si nota l'influenza di Joseph Conrad, che potrebbe aver ispirato le atmosfere esotiche, ma anche lo studio psicologico dei personaggi. Corto Maltese potrebbe essere, secondo Umberto Eco, "un Lord Jim con tutte le caratteristiche, i rimorsi e le ambiguità, meno la sconfitta, insomma un Lord Jim a cui è andata bene". ${ }^{26}$ In Una ballata del mare salato il gioco delle citazioni comincia già nel titolo, che richiama la poesia di S.T. Coleridge The Rime of the Ancient Mariner, in italiano La ballata del vecchio marinaio, e preannuncia il tono lirico e corale del fumetto. Come nel poema di Coleridge, il mare fa qui da sfondo alla narrazione non come spazio fisico, ma come spazio immaginario, cornice mitica in cui spazio e tempo si dilatano in un'atmosfera fiabesca :

Cain e Pandora vengono catturati da Rasputin il primo novembre 1913, ma tutti arrivano all'Escondida dopo il 4 agosto 1914 (il Monaco li informa che in quella data 
è scoppiata la guerra) e grosso modo tra il settembre e l'ultima decade di ottobre, quando entrano in scena gli inglesi. Tra due pagine di Coleridge e due discussioni con Slütter, è passato un anno, nel corso del quale il sottomarino si muoveva per vaghe rotte, con l'indolenza curiosa, la sete di deriva dei bucanieri del XVII secolo, del Vecchio Marinaio, e del capitano Ahab [...]. Ed è in questa bruma che affetta spazio e tempo che nascono i miti. ${ }^{27}$ effetti tutti i protagonisti dei fumetti di Hugo Pratt amano leggere, in una sorta di gara di erudizione :

a un certo punto Pandora appare dolcemente appoggiata all'opera omnia di Melville, e Cain legge Coleridge, autore di un'altra ballata, quella del 'Vecchio Marinaio'. Tra l'altro la legge in traduzione italiana e la trova, con Melville, a bordo di un sottomarino tedesco (fa parte della biblioteca di slütter, che lascerà a Escondida, dopo la sua morte, anche un Rilke e uno Shelley; Cain peraltro in chiusura citerà Euripide). ${ }^{28}$

Persino lo spietato Rasputin legge Louis Antoine de Bougainville e conosce Platone..$^{29} \mathrm{Le}$ dinamiche che si stabiliscono tra i fumetti di Hugo Pratt e le opere che cita, sono di varia natura. Il più delle volte sono da intendersi come degli omaggi che il padre di Corto Maltese dedica agli autori, personaggi o opere che ha amato. Non manca inoltre la volontà di sfruttare quella che, secondo Andrea Bernardelli, "è la principale funzione della citazione, [...] l'invocazione di autorità". ${ }^{30}$ Nell'appellarsi all' auctoritas di autori e opere spesso canonici e prestigiosi, Hugo Pratt compie un tentativo di alzare lo status del fumetto, avvicinandolo a media più prestigiosi, come la letteratura. Altre volte il rimando ad altri testi è così insistente che diventa esagerato, quasi grottesco e quindi ironico : "lo fa persino con una strizzata d'occhio post-moderna, come si vede ad esempio quando, anziché subire un'influenza, la ostenta con vezzo citazionistico". ${ }^{31}$

Una strategia spesso utilizzata da Hugo Pratt è quella di accostare riferimenti a opere o figure provenienti da contesti completamente diversi oppure appartenenti a codici distinti, che suggerisce al lettore la sensazione della presenza di uno scarto ironico tra $\mathrm{i}$ testi evocati. Questo uso ironico della citazione sfocia nella parodia che è, secondo un'accezione "moderna o postmoderna, [...] una sorta di confronto dialettico tra i distinti codici dei testi coinvolti". ${ }^{32}$ In questo senso tutta l'opera di Hugo Pratt può essere letta come un tentativo di scardinare i pregiudizi verso la cultura cosiddetta popolare. Pratt mescola continuamente le carte intrecciando cultura alta e fonti popolari. Mentre i personaggi dei suoi fumetti preferiscono i classici e fanno bella mostra dei loro volumi (Thomas More, Coleridge, Melville, ecc.), dal testo trapelano continui riferimenti a opere meno canoniche come fumetti, romanzi e i film di avventura. Pratt ha sempre rivendicato con orgoglio le sue letture meno nobili. In una nota introduttiva all'edizione Albatros del 1976 di Una ballata del mare salato dedica il suo fumetto a un scrittore irlandese, Henry de Vere Stacpoole, autore di La laguna azzurra: "fu questo scrittore, e non Robert Louis Stevenson, non Conrad e neppure Melville, che per primo mi fece amare i mari del sud. È a lui che dedico questa ballata".

Tra le fonti popolari di Pratt c'è ovviamente il fumetto. Su tutti un autore come Milton Caniff, autore di classici del fumetto d'avventura come Terry and the Pirates e Steve Canyon. Grande fonte d'ispirazione per Pratt fu anche il cinema, quello degli anni cinquanta e sessanta dei grandi film di avventura hollywoodiani. Pratt è stato un assiduo frequentatore delle sale cinematografiche e molte delle storie che ha visto sul grande schermo sono filtrate poi nei suoi fumetti. La scena in cui si vede Corto Maltese legato ad 
una zattera (figura 6), per esempio, "vuole essere un'allusione a una sequenza di Wake of the Red Witch, un film di pirati di Edward Ludwig con John Wayne e Gail Russell". ${ }^{33}$ Nella figura 7, Hugo Pratt accosta in modo evidentemente ironico la figura del cavaliere Klingsor - personaggio del Parzival di Wolfram von Eschenbach e di Richard Wagner, nonché personaggio di un romanzo di Hermann Hesse, a King Kong, il popolare scimmione del cinema.

Figura 7

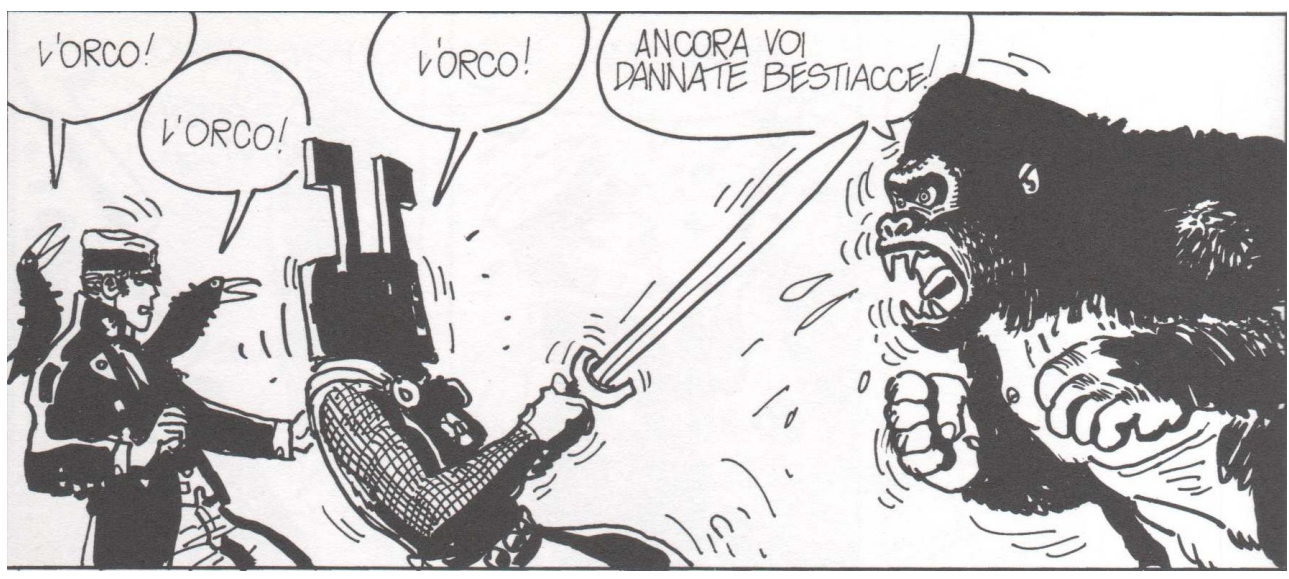

Le elvetiche- Rosa alchemica

\section{Uno spazio ibrido}

La saga di Corto Maltese rappresenta, a mio avviso, un continuo tendere verso la letteratura, sia da un punto di vista contenutistico, sia per quanto riguarda la forma editoriale. I fumetti di Hugo Pratt sono stati pubblicati su supporti sempre più autorevoli e letterari: dalle prime edizioni sui giornali per ragazzi sono stati stampati poi in riviste più prestigiose, per arrivare infine alle edizioni in volume. Nei suoi racconti Hugo Pratt ha da sempre messo in atto strategie narrative tipiche del romanzo come la profonda introspezione dei personaggi e il complesso e articolato intreccio narrativo, rispecchiandosi continuamente nel mondo della letteratura tramite allusioni o citazioni. Anche dal punto di vista del disegno l'evoluzione di Hugo Pratt è costante: dalla linea nervosa e carica di Una ballata del mare salato, al tratto deciso e quasi sbrigativo di $\mathrm{Mu}$, la differenza è notevole. Sembra quasi che nella ultima fase della sua vita mirasse a una maggiore iconicità, a una sempre maggiore sintesi del disegno, concentrandosi maggiormente sullo sviluppo narrativo: "mano a mano che passano gli anni, Pratt sembra frettoloso nei confronti del disegno e, per converso, sempre più attento alla parte 'scritta' dei suoi fumetti, alla vera e propria narrazione". ${ }^{34}$

Se da una parte l'autore di Corto Maltese ha di fatto contribuito ad accreditare il fumetto come prodotto culturalmente rilevante, dall'altra egli ha sempre ribadito con fierezza le origini " povere » della sua arte. Hugo Pratt non amava definirsi un artista; con una punta di sarcasmo egli preferiva il termine "fumettaro": "Sì, fumettaro. Perchè è una brutta parola fumettaro. Voglio essere un fumettaro, perchè fumettaro forse è un'offesa. I miei colleghi preferiscono essere chiamati illustratori, disegnatori, grafici, altre cose, ma io penso che invece al giorno d'oggi, essere un operatore del mondo della fantasia, come lo può essere un fumettaro, è ancora una cosa importante". ${ }^{35}$ Tutta l'opera di Hugo Pratt 
può essere vista quindi come un continuo tentativo di scardinare le gerarchie culturali. Attraverso l'accostamento ironico di registri culturali diversi, mettendo sullo stesso piano cinema, poesia, letteratura d'avventura e fumetto, Pratt mirava ad annullare le distanze tra le sfere culturali alte e basse. La saga di Corto Maltese sembra insomma voler mettere in crisi il sistema dei valori culturali, evidenziando con ironia i limiti di una netta separazione tra cultura alta e popolare. La ricezione dell'opera di Hugo Pratt sembra confermare la doppia natura della sua opera. Le avventure di Corto Maltese hanno incuriosito alcuni critici letterari e alcuni intellettuali per la sottigliezza delle soluzioni narrative, tanto che a Umberto Eco viene attribuita questa sentenza: "Quando ho voglia di rilassarmi leggo un saggio di Engels, se invece desidero impegnarmi leggo Corto Maltese". ${ }^{36}$ Allo stesso tempo Corto Maltese non ha perso il suo fascino popolare e viene continuamente apprezzato da moltissimi lettori e semplici appassionati di fumetto. Hugo Pratt è stato anche uno dei primi autori di fumetto a essere considerato come vero e proprio artista. I suoi fumetti sono stati esposti in numerosi musei, a cominciare dalla grande esposizione al Grand Palais di Parigi nel 1986. Oggi i suoi disegni, acquerelli, assieme ai volumi con le prime edizioni dei suoi fumetti, sono diventati oggetto di culto tra i collezionisti e vengono battuti dalle case d'asta a prezzi esorbitanti. ${ }^{37}$

Le iniziative riguardanti il marinaio di Pratt non si limitano però ai musei e alle élite culturali. Corto Maltese è diventato un brand di successo da sfruttare a fini commerciali, con esiti a volte apprezzabili, a volte meno. L'immagine di Corto è stata associata, ad esempio, ad un'ottima guida turistica alla città di Venezia, realizzata da due storici collaboratori di Hugo Pratt. ${ }^{38} \mathrm{Nel} 2002$ una produzione italo-francese ha realizzato una serie animata dei fumetti di Corto Maltese producendo alcuni lungometraggi e dei cortometraggi ${ }^{39}$ Infine in commercio è possibile trovare ogni sorta di oggetto con il marchio di Corto Maltese. Si va dal posacenere, all'astuccio porta-tabacco ed esiste anche una collezione di abiti ispirati all'abbigliamento del marinaio nato a Malta. I fumetti di Hugo Pratt navigano quindi in uno spazio ibrido, dove la distinzione tra alto e popolare si perde in un'atmosfera di indefinitezza, intrappolata nelle nebbie dell'oceano Pacifico. Il capitano Corto Maltese rimane una figura altrettanto ibrida, a metà strada tra un Ancient Mariner e un Burt Lancaster, tra un Lord Jim e un Tarzan.

\section{NOTE}

1. Per una bibliografia completa dell'opera di Pratt rimando al sito Les Archives Hugo Pratt http:// www.archivespratt.net/ (visitato il 23/07/2014).

2. Cfr. Alice Favaro: Hugo Pratt: un emigrante de la historieta. Experiencia y formación leteraria en Argentina. In: Dal realismo magico al fumetto. Problemi di letterature comparate. A cura di Alessadro Scarsella. Venezia: Granviale, 2013, pp. 77-91.

3. Luigi F. Bona: Appunti sulle origini e sulla storia del fumetto italiano. Fondazione Franco Fossati. url: http://www.lfb.it/fff/fumetto/storia/st_it/st_it_010.htm (visitato il 22/07/2014).

4. Ibid.

5. Ibid.

6. Giovanni Gandini: Editoriale. In: Linus 1 (apr. 1965), p. 01. 
7. Umberto Eco, Oreste Del Buono e Elio Vittorini: Charlie Brown e i fumetti. In: Linus 1 (apr. 1965), p. 02 .

8. Giuseppe Peruzzo: Persone di Nuvola. Le riviste di fumetti d'autore. Torino: Q Press, 2003, p. 117.

9. Il termine è molto vago e tutt'oggi ancora molto discusso dalla critica. Cfr. Dietrich Grünewald: Struktur und Geschichte der Comics. Beiträge zur Comicforschung. Essen: Ch. A. Bachmann Verlag, 2010 e anche Vittorio Spinazzola: Tirature 2012. Graphic novel. L'età adulta del fumetto. Milano: Il Saggiatore, 2012.

10. Leonardo Bizzarro: Hugo Pratt e Corto Maltese gemelli diversi dell'avventura. In: La Repubblica (10 ago. 2012). url: http://torino.repubblica.it/cronaca/2012/08/10/news/ hugo_pratt_e_corto_maltese_gemelli_diversi_dell_avventura-40670200/ (visitato il 22/07/2014).

11. http://www.corriere.it/cultura/12_luglio_09/corto-maltese-una-ballata-del-maresalato_afe5035a-c997-11e1-826a-3168e25ab050.shtml (visitato il 22/07/2014).

12. La serie comprende 14 uscite a colori. url: http://www.rizzoli-lizard.com/corto-maltese-dihugo-pratt-la-serie-digitale/ (visitato il 06/10/2014).

13. Claudio Gallo e Giuseppe Bonomi: Tutto cominciò con Bilbolbul... Per una storia del fumetto italiano . Verona: Perosini, 2006, p. 121.

14. Luca Raffaelli: Tratti\&Ritratti. I grandi personaggi del fumetto da Alan Ford a Zagor. Roma: Minimum Fax, 2009, p. 81.

15. Gianni Brunoro: Corto Come un romanzo. Illazioni su Corto Maltese ultimo eroe romantico. Roma: Lizard, 2008, p. 26.

16. Luca Boschi: Irripetibili. Le grandi stagioni del fumetto italiano. Roma: Coniglio Editore, 2007, p. 46 .

17. Hugo Pratt e Dominique Petitfaux: Il desiderio di essere inutile. Ricordi e riflessioni. Roma: Lizard, 1996, p. 273.

18. Raffaelli, cit., p. 82.

19. "All Europe contributed to the making of Kurtz". Joseph Conrad: Heart of Darkness and Other Tales. London: William Pickering, 1993, p. 54.

20. Pratt e Petitfaux, cit., p. 274.

21. Ibid.

22. Herbert Heinzelmann: Corto im "anderen Zustand". Aspekte der Moderne und Postmoderne in Hugo Pratts Südseeballade und im Maltese-Zyklus. In: Comics, Mangas, Graphic Novels. A cura di H.L. Arnold e A.C. Knigge. Text + Kritik: Sonderband. München: Edition Text + Kritik, 2009, pp. 109-124, p. 111.

23. Roberto Ellero: E Corto Maltese disse Vive la France. In: La Repubblica (21 gen. 1986). url: http:// ricerca.repubblica.it/repubblica/archivio/repubblica/1986/01/21/corto-maltese-disse-vive-lafrance.html (visitato il 22/07/2014).

24. Giovanni Marchese: Leggere Hugo Pratt. L'autore di Corto maltese tra fumetto e letteratura. Latina: Tunué, 2006, p. 8.

25. Cfr. Gérard Genette: Palinsesti. Trad. da Raffaella Novità. Torino: Einaudi, 1997.

26. Vincenzo Mollica, cur. Dedicated to Corto Maltese. Montepulciano: Editori del Grifo, 1981, p. 52.

27. Umberto Eco: Geografia imperfetta di Corto Maltese. In: Tra menzogna e ironia. Milano: Bompiani, 1998, pp. 99-107, p. 104.

28. Ibid., pp. 99-100.

29. Nella versione animata di Una ballata del mare salato si esagera nel far apparire Rasputin come uomo di cultura. In una scena, di cui non vi è traccia nel fumetto, lo si sente citare a braccio dei versi di Rainer Maria Rilke. In realtà Rasputin rimane un personaggio rude, che legge soprattutto per interesse pratico. In $\mathrm{Mu}$, esasperato dal continuo parlar colto di Corto Maltese, egli si arrabbia e lo minaccia: "Non ricominciamo con le tue erudizioni".

30. Andrea Bernardelli: Intertestualità. Milano: La Nuova Italia, 2000, p. 32. 
31. Umberto Eco: All'ultima storia capi: Corto Maltese sono io. In: La Repubblica (7 ago. 2005). url: http://www.repubblica.it/2005/h/sezioni/spettacoli_e_cultura/ecopratt/ecopratt/ecopratt.html (visitato il 22/07/2014).

32. Bernardelli, cit., p. 75.

33. Pratt e Petitfaux, loc. cit.

34. Brunoro, cit., p. 285.

35. Valerio Corzani: Passioni: Corto Maltese e altre latitudini. Quarta puntata. In: Radio 3 (24 giu. 2012). url: http : / / www . radio3 . rai . it / dl / radio3 / programmi / archivio / ContentSet-5e5eaec7-9e54-479f-b615-bb03446be4de.html (visitato il 22/07/2014).

36. Dominique Petitfaux: All'ombra di Corto. Milano: Rizzoli, 1992, p. 166.

37. Bizzarro, cit.

38. Guido Fuga e Lele Vianello: Corto Sconto. Itinerari fantastici e nascosti di Corto Maltese a Venezia. Roma: Lizard, 1998.

39. Cfr. Pierfilippo Dionisio: Corto Maltese dal fumetto all'animazione. UBCfumetti. url: http:// www.ubcfumetti.com/mag/cortomaltese.htm (visitato il 22/07/2014).

\section{RIASSUNTI}

At the time of his first appearance in 1967 the Corto Maltese comics marked a turning point in the world of comics : the complexity of its protagonist and the subtlety of the plot were revolutionary. They were indeed "literary" comics : its author Hugo Pratt declared he was aiming to create "letteratura disegnata" - drawn literature. Corto Maltese was at first published in comic-magazines for children like Corriere dei Piccoli, then it moved to a new type of magazine that was more adult-oriented and had intellectual ambitions, like Linus. Finally Una ballata del mare salato, Corto Maltese's first adventure, was re-published in 1972 in book form by Mondadori, a major publishing house. Corto Maltese's literary qualities and its publication history are two innovative aspects of these comics, which elevate them from the status of "popular literature" to achieve "cultural" acknowledgement. At the same time, Hugo Pratt was a great consumer of "popular" fiction, comics and adventure/action movies, which he used as sources of inspiration for his comics. He was a fierce defender of the "popular" origins of his work and refused to call himself an "artist". This essay investigates these two apparently opposed aspects of the Corto Maltese comics. I will argue that Hugo Pratt aimed to overcome the "cultural" gap between the genres and the different media and to demolish the wall between "high" and "popular" culture. 